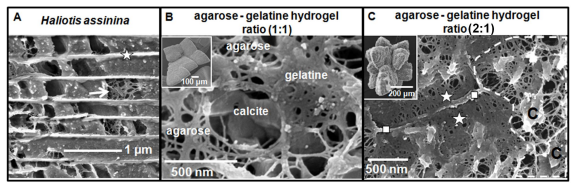

Figure 1. Characteristics of extracellular carbonate hard tissue (1A) and hydrogel (1B, 1C) matrices.

Keywords: EBSD, calcite-hydrogel aggregate, extracellular matrix, gelatin, agarose

\section{MS14-04 Polar ordering of macromolecular chains in biomimetic composite materials and natural tissues

\author{
Martin Sommer ${ }^{1}$, Matthias Burgener ${ }^{1}$, Hanane Aboulfadl ${ }^{1}$, Jürg
} Hulliger $^{1}$}

1. Department of Chemistry and Biochemistry, University of Bern, Freiestrasse 3, CH-3012 Bern, Switzerland

email: martin.sommer@dcb.unibe.ch

The state of alignment of macromolecules in biomimetic materials and natural tissues will be discussed by investigating a mechanism of electrical polarity formation: An in vitro grown biomimetic fluorapatite (FAp)/ gelatin composite is analyzed for its polar properties by second harmonic (SHGM) and scanning pyroelectric microscopy (SPEM). Growth media containing biological macromolecules do not only influence the morphology, but can also have a significant impact on the polarity of a composite [1]. Hexagonal prismatic seed crystals of FAp formed in gelatin represent a monodomain polar state due to aligned mineralized gelatin molecules [1]. Later growth stages, expressing a dumbbell morphology, develop into a bipolar state because of surface recognition by gelatin functionality.

Other inorganic materials like $\mathrm{CaCO}_{3}$ (calcite), $\mathrm{CaSO}_{4}$ and $\mathrm{CaC}_{2} \mathrm{O}_{4}$ formed analogous composites when grown in a gelatin matrix. In addition, all of these composites revealed a similar behavior regarding polarity formation as compared to FAp. Subsequently the gelatin matrix was replaced by other gels, such as agar-agar and carrageenan, which developed the same kind of bipolar state. By growing the inorganic components in tetramethylorthosilicate (TMOS, nonpolar gel), SPEM experiments did not reveal any polarity. In all grown composites, the only present organic and polar material are the polar gel macromolecules. Single crystals of the investigated inorganic components, e.g. FAp and $\mathrm{CaCO}_{3}$, are centrosymmetric. Therefore, the only possible origin of polarity in these biomimetic composites is due to the incorporated macromolecules.

Comparing SPEM data of natural hard tissues (teeth and bone) with biomimetic FAp/ gelatin, calcite/ gelatin and other investigated composites, a surprising analogy in view of growth-induced states of polarity is found: The development of polarity in vivo and in vitro can be explained by a Markov-type mechanism of molecular recognition during the attachment of macromolecules. Furthermore, SHGM was able to reveal the polar structure of tissues by the application of phase sensitive experiments and the use of a polar reference crystal [2].

[1] Burgener, M.; Putzeys, T.; Gasthi, M. P.; Busch, S.; Aboulfadl, H.; Wübbenhorst, M.; Kniep, R.; Hulliger, J. Biomacromolecules 2015, 16, 2814-2819.

[2] Aboulfadl, H.; Hulliger, J. J. Struct. Biol. 2015, $192,67-75$.

Keywords: polarity, polarity formation, biomimetic, composite materials, natural tissues 